\title{
Effectiveness of third-generation chemotherapy on the survival of patients with advanced non-small cell lung cancer in Norway: a national study
}

\author{
C von Plessen, ${ }^{1,2,3}$ T-E Strand, ${ }^{4}$ T Wentzel-Larsen, ${ }^{5}$ E Omenaas, ${ }^{2,5} \mathrm{~N}$ Wilking, ${ }^{6}$ \\ S Sundstrøm, ${ }^{3,7}$ S Sörenson ${ }^{2,8}$
}

${ }^{1}$ Department of Thoracic Medicine, Haukeland University Hospital, Bergen, Norway; ${ }^{2}$ Institute of Medicine, University of Bergen, Norway; ${ }^{3}$ Norwegian Lung Cancer Study Group; ${ }^{4}$ Cancer Registry of Norway, Oslo, Norway: ${ }^{5}$ Centre for Clinical Research, Haukeland University Hospital, Bergen,

Norway; ${ }^{6}$ Karolinska Institute,

Stockholm, Sweden;

7 Department of Oncology, St Olav's University Hospital, Trondheim, Norway:

${ }^{8}$ Department of Pulmonary Medicine, Linköping University Hospital and University of Linköping, Sweden

Correspondence to: Dr C von Plessen, Department of Thoracic Medicine, Haukeland University Hospital, N-5021 Bergen, Norway; christian.von.plessen@ helse-bergen.no

Received 12 November 2007 Accepted 11 March 2008 Published Online First 4 April 2008

\section{ABSTRACT}

Background: To investigate whether the introduction of modern third-generation chemotherapy was associated with survival benefits in a national population of patients with advanced non-small cell lung cancer (ANSCLC) and to explore geographical and temporary variations in the utilisation of chemotherapy.

Methods: All patients with ANSCLC in the Cancer Registry of Norway during 1994-2005 were included. Using sales of vinorelbine as an indicator for chemotherapy, annual county utilisation rates were calculated. Survival before and after the general introduction of vinorelbine and associations between survival and variations in utilisation in counties were investigated. In a subgroup, the predictors of having received chemotherapy were explored.

Results: Of 24875 registered patients with lung cancer, 13757 had ANSCLC. The annual utilisation of the indicator drug in Norway increased from 3.7 to $184.2 \mathrm{~g}$ (1998-2005). Median survival increased from 149 to176 days $(p<0.001)$. The adjusted hazard ratio (HR) for a diagnosis after the introduction was $0.93(95 \% \mathrm{Cl} 0.88$ to 0.99 ). County utilisation rates of vinorelbine (increments of $100 \mathrm{mg} / 1000$ inhabitants) were inversely associated with the risk of death (HR $0.84,95 \% \mathrm{Cl} 0.73$ to 0.98). County of residence predicted chemotherapy utilisation with odds ratios in the range $0.13195 \% \mathrm{Cl} 0.1$ to 0.19$)$ to $1.04(95 \% \mathrm{Cl} 0.64$ to 1.69$)$, a county with traditionally high utilisation as reference.

Conclusion: Utilisation of third-generation chemotherapy was associated with slightly increased survival of patients with ANSCLC. Geographical and temporal differences in utilisation indicate variable quality of delivered care.

Lung cancer is the third most common mortal disease in industrialised countries, taking 350000 lives in Europe ${ }^{1}$ and 160000 in the United States ${ }^{2}$ annually. The prognosis has improved slightly during the past few decades. Survival varies across countries, however, with registry-based relative one-year survival rates of, for example, $25 \%$ in England $^{3}$ and $36 \%$ in Sweden. ${ }^{4}$

Approximately $60 \%$ of patients with non-small cell lung cancer are being diagnosed with regionally advanced or metastatic disease, which in most cases implies a palliative treatment intention. The advanced stages are often subsumed under the term "advanced non-small-cell lung cancer" (ANSCLC), which covers TNM stages IIIB and IV. In this situation, chemotherapy, usually a platinum compound and one of the third-generation drugs vinorelbine, gemcitabine, docetaxel, or paclitaxel, , $^{5-7}$ is given to prolong survival and improve quality of life (QoL). In patients with good performance status (PS), chemotherapy increases one-year survival to up to $40 \%$ from $10 \%$ with supportive care alone, prolongs survival by approximately 3 months without detriment to ${ }^{8}$ or with partial improvement of QoL..$^{9-13}$ On the other hand, patients experience side effects, spend time travelling and waiting and only approximately $20 \%$ will achieve an objective treatment response. ${ }^{14}$

The effects of chemotherapy are amply documented in the selected populations of formal clinical trials, but whether favourable outcomes can be achieved in the unselected patient populations of everyday clinical practice is disputed. Vardy et $a l^{15}$ found that only one third of patients attending an oncology referral centre met the common inclusion criteria of chemotherapy protocols. In particular, elderly patients are often underrepresented in clinical trials ${ }^{16} 17$ and the proportion of patients with unfavourable prognostic factors such as PS 2, TNM stage IV, and weight loss in phase III trials of chemotherapy has even declined during the past few decades. ${ }^{18} 19$

In addition to patient and disease-related factors, the performance of the healthcare system influences the outcomes of patient populations. This performance is currently ubiquitously hampered by unwarranted geographical variations in medical practice. ${ }^{20}$ Unexplained variations in the use of chemotherapy for lung cancer, ${ }^{21}$ the underuse of effective care ${ }^{22} 23$ as well as the potential overuse of supply-sensitive care for lung cancer ${ }^{24}$ have been observed. In the community setting in the United States during the second half of the 1990s, only approximately $30 \%$ of patients over 65 years of age received chemotherapy, although chemotherapy use was clearly associated with prolonged survival. ${ }^{25}{ }^{26}$ Cartman et a ${ }^{27}$ found that variations in the combined treatment rates of surgery, radiotherapy and chemotherapy in the districts of a region in England were associated with the survival of patients with lung cancer. To our knowledge, however, associations between variations in the use of chemotherapy alone and survival have not been studied previously in a national population of patients with ANSCLC.

The aim of the current study was to explore geographical and year to year variations in the use of chemotherapy for ANSCLC and to test the hypothesis that the utilisation of modern thirdgeneration chemotherapy was associated with survival benefits for patients with ANSCLC in 
Norway. Norway has a population of 4.7 million and approximately 2300 new cases of lung cancer annually. Chemotherapy against ANSCLC was introduced around 1998. We used data from the national Cancer Registry of Norway (CRN), the Norwegian Institute of Public Health, patient administrative systems and hospital pharmacies.

\section{METHODS}

We included all patients with lung cancer in Norway reported to CRN during 1994-2005. The period comprised 4 years before and 7 years after the general introduction of chemotherapy for ANSCLC in Norway.

CRN covers the Norwegian population as a whole. Reporting to the registry is mandatory by law since 1952. Incomplete clinical reports are supplemented with information from hospital files. Since 1998, all Norwegian hospitals have submitted electronic discharge summaries of patient administrative data, including procedure codes for ambulatory chemotherapy. Furthermore, since 1993 hospitals have reported radiotherapy to the registry. The Cause of Death Registry at Statistics Norway supplies death certificates. CRN uses a condensed TNM staging system in which $\mathrm{T} \leqslant 2, \mathrm{~N} 0$ and $\mathrm{M} 0$ are coded as "localised", all $\mathrm{T} \geqslant 3, \mathrm{~N} \geqslant 1$ and $\mathrm{M} 0$ as "regionally advanced" and any $\mathrm{M}$ as "metastatic" disease.

We regarded patients with clinically staged regionally advanced or metastatic non-small-cell lung cancer to be candidates for palliative chemotherapy and included them in the study. We excluded patients with two or more diagnoses of lung cancer, post mortem diagnosis, registered emigration or

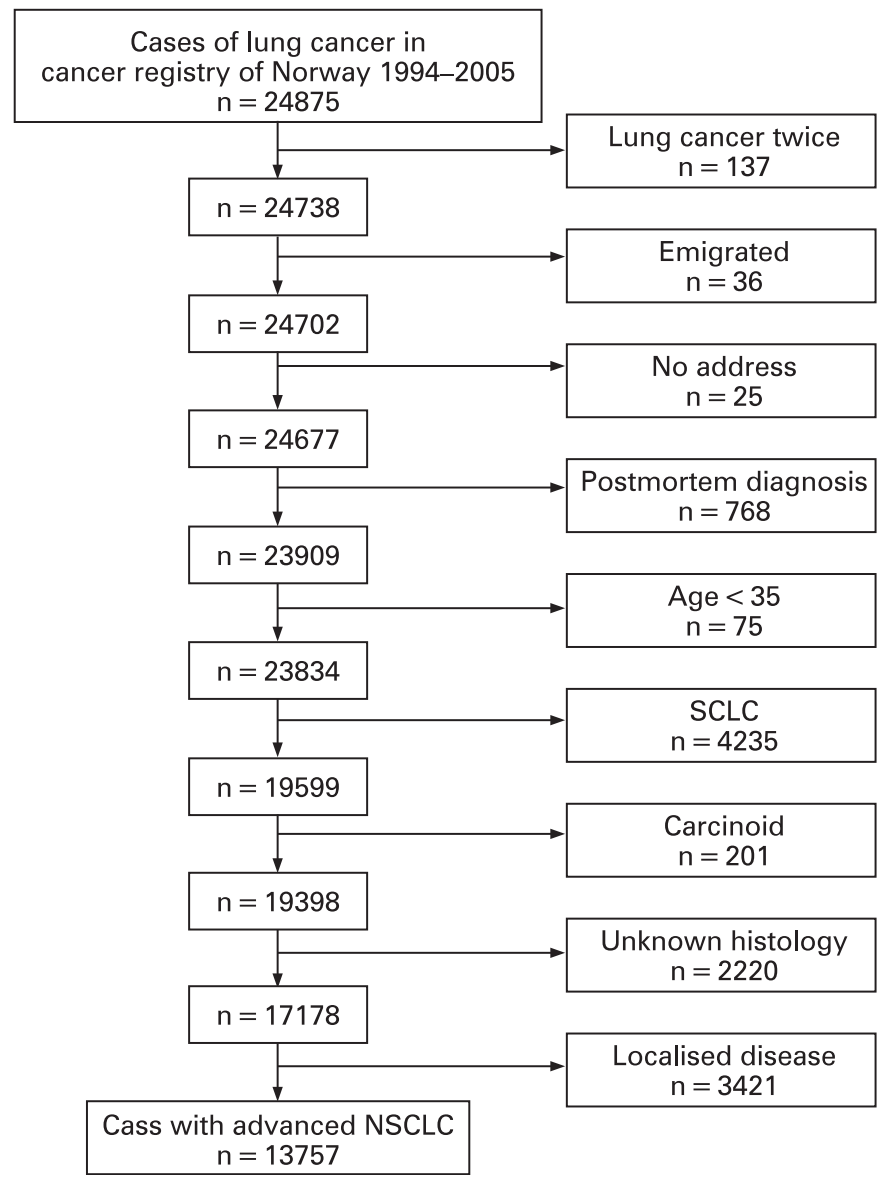

Figure 1 Patients in the study. NSCLC, non-small-cell lung cancer; SCLC, small-cell lung cancer. untraceable address, small-cell, carcinoid or unknown histology. Furthermore, as a result of legal regulations regarding the risk of personal identification we excluded cases that were diagnosed under the age of 35 years $(<1 \%)$.

\section{Third-generation chemotherapy}

Norway had 19 counties and up to 62 hospitals during the study period. Every county had at least one hospital in which lung cancer patients received chemotherapy. In a telephone survey of all hospitals, we found that 52 hospitals had treated patients with lung cancer during the study period. At five hospitals patients with ANSCLC were exclusively treated at departments of respiratory diseases. We obtained sales of vinorelbine and gemcitabine from the pharmacies at these hospitals of, respectively, the respiratory and other departments, and thus calculated their use for ANSCLC versus other types of cancer.

The Norwegian Institute of Public Health registers sales of cytotoxic drugs in grams per year and county, according to reports from the pharmaceutical industry. We chose vinorelbine as the indicator drug for chemotherapy utilisation in Norway because it is registered for non-small-cell lung cancer and breast cancer only. The latter patients received vinorelbine rarely in routine clinical practice and only occasionally within third-line protocols. Internationally, gemcitabine is commonly used to treat patient with ANSCLC, but in Norway during the study period it was used within two national protocols and rarely in routine practice and could thus serve as a comparator to vinorelbine. Cisplatin, carboplatin, doxorubicin, epirubicin, vincristine, cyclophosphamide, etoposide, docetaxel and paclitaxel were not suitable indicators because they had too many indications, were not part of third-generation regimens, or were reserved for second-line treatment.

We calculated yearly rates of vinorelbine, gemcitabine, carboplatin and cisplatin utilisation per county. We defined the unit of vinorelbine utilisation as $100 \mathrm{mg} / 1000$ inhabitants or $100 \mathrm{mg} /$ patient with non-small-cell lung cancer. The units of the other drugs were $1000 \mathrm{mg} / 1000$ inhabitants or $1000 \mathrm{mg} /$ patient to account for the higher standard doses. We assumed that chemotherapy sales and use were equal.

In addition, in a subgroup we estimated the geographical variation in chemotherapy utilisation in Norway from registered International Classification of Diseases procedure codes for ambulatory chemotherapy (Z51.1).

\section{Analyses and statistics}

We carried out four analyses. First, we compared survival time before (1994-7) and after (2000-5) the general introduction of

Table 1 Characteristics of 13757 patients with advanced non-small-cell lung cancer diagnosed during 1994-2005

\begin{tabular}{ll}
\hline Patient characteristics & $\%$ \\
\hline Mean age (years) & 67.5 \\
Men & 65.1 \\
Squamous cell carcinoma & 28.5 \\
Adenocarcinoma & 38.0 \\
Non-specified NSCLC & 26.5 \\
Large-cell carcinoma & 7.1 \\
Condensed TNM & \\
$\quad$ Regionally advanced & 27.5 \\
$\quad$ Metastatic & 72.6 \\
\hline
\end{tabular}

NSCLC, non-small-cell lung cancer; TNM, tumour mode metastasis. 
chemotherapy for ANSCLC in a Kaplan-Meier analysis applying the log rank test. In this we excluded patients diagnosed during a transition period of 2 years (1998-9). Adjustment variables in the corresponding Cox regression were patients' age at diagnosis (10-year increments), gender, having undergone surgery with curative intention or thoracic radiotherapy and utilisation rates of cisplatin and carboplatin. Second, we investigated associations between survival and the county utilisation rate of the indicator drug vinorelbine in a Cox regression. In this analysis, we added gemcitabine to the adjustment variables mentioned above. Third, in a subgroup we compared the survival of patients with or without a registered chemotherapy course in a Kaplan-Meier analysis and a Cox regression. In the latter we adjusted for age, gender, year of diagnosis and county of residence. In the same subgroup we explored predictors of having received chemotherapy in a logistic regression. Adjustment variables were again age, gender, year of diagnosis, surgery and radiotherapy. We defined statistical significance for all analyses as $p<0.05$ and carried through all analyses with the $R$ statistics software. ${ }^{28}$

\section{RESULTS}

From 1994 to 2005, 24875 patients were diagnosed with lung cancer in Norway, of these 13757 met the study criteria (fig 1). The percentage of patients with ANSCLC increased from an annual average of 72.5 to 84.8 during 1994-7 and 2000-5, respectively.

Patient characteristics are given in table 1. Patients' mean age at diagnosis increased from 67 years (SD 10.37) during 19942000 to 68 years (SD 10.3) during 2001-5. Respectively, 759 and 347 patients had survived 3 and 5 years, most of them (66\% and $73.5 \%$ ) had been operated with a curative intention.

\section{Utilisation of third-generation chemotherapy in Norway}

In 1998, the first year after its registration in Norway, vinorelbine was prescribed in one of the 19 counties, whereas in 2003 the drug was used in all counties. Respectively, $3.7 \mathrm{~g}$ and $184.2 \mathrm{~g}$ of

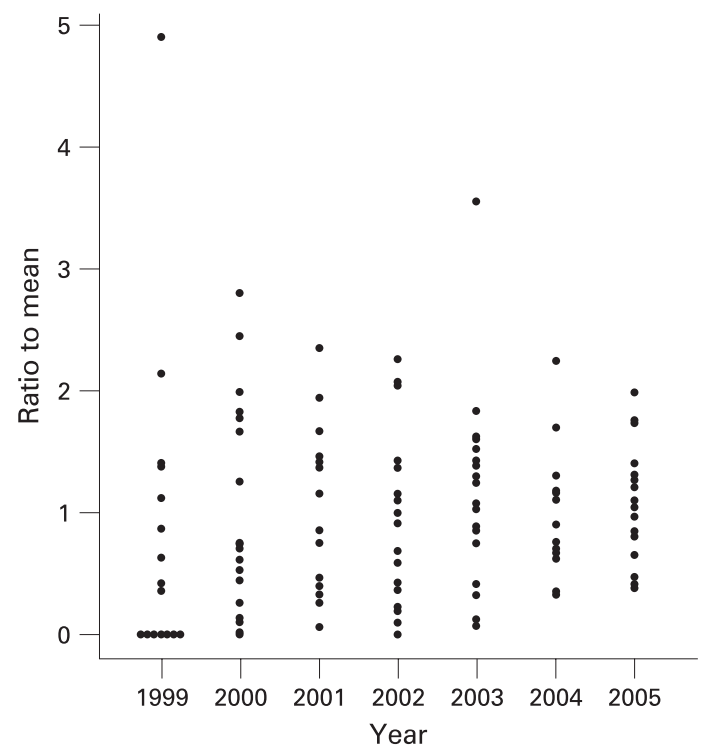

Figure 2 Distribution of the utilisation of the indicator drug vinorelbine in the Norwegian counties ( $N=19$, some dots represent two counties). Each dot represents the ratio of the utilisation in one county to the mean utilisation in Norway. vinorelbine were sold in 1998 and 2005. The corresponding number of treated patients is 15 and 723 , estimated from three cycles of vinorelbine with a dose of $42.5 \mathrm{mg}$ given to a patient, of $60 \mathrm{~kg}$ weight and $170 \mathrm{~cm}$ height, at days 1 and 8 . Differences in the utilisation of vinorelbine across counties diminished gradually. Whereas the ratio of the utilisation in one county to the mean utilisation in Norway had a range between the highest and lowest user of 4.9 in 1999, it was 1.6 in 2005 (fig 2).

On average, $88.2 \%$ of vinorelbine and $44.2 \%$ of gemcitabine was sold to departments exclusively treating patients with respiratory diseases at five hospitals, each located in one of the five Norwegian health regions.

\section{Survival before and after the introduction of third-generation chemotherapy}

Median survival was 149 and 176 days during 1994-7 and 20005 , respectively (log-rank $\mathrm{p}<0.001$ ) and one-year survival increased from $23.8 \%$ to $27.9 \%$. The corresponding adjusted hazard ratio (HR) was 0.93 (95\% CI 0.88 to 0.99$)$ in favour of a diagnosis during the latter period.

\section{Survival in Norwegian counties and utilisation of third- generation chemotherapy}

Utilisation rates of vinorelbine $(100 \mathrm{mg}$ per 1000 county inhabitants) were inversely associated with the risk of death with an adjusted HR of 0.84 (95\% CI 0.73 to 0.98$)$. We found a weaker association for gemcitabine (1000 mg per 1000 county inhabitants, adjusted HR 0.98, 95\% CI 0.98 to 1.01), which had rarely been used against lung cancer in Norway. The adjusted HR for the utilisation of vinorelbine in $100 \mathrm{mg}$ per ANSCLC patient in a county were 0.94 (95\% CI 0.90 to 0.99 ) and for gemcitabine in $1000 \mathrm{mg}$ per ANSCLC patient 0.99 (95\% CI 0.98 to 1.01). In an exploratory analysis using splines, ${ }^{29}$ we did not find non-linearity in vinorelbine use $(p=0.67)$.

\section{Survival of individual patients and registered chemotherapy procedure codes and predictors of chemotherapy utilisation in Norway}

A total of 8850 cases were diagnosed with ANSCLC during 1999-2005, the time period of the registration of ambulatory chemotherapy in Norway. Of these, 4303 patients (50.3\%) had at least one course of chemotherapy registered by an ambulatory procedure code. One-year and median survival with and without chemotherapy were $33.8 \%$ and 241 days and $22.4 \%$ and 104 days, respectively (log rank $\mathrm{p}<0.001)$. The corresponding HR was 0.63 (95\% CI 0.60 to 0.66$)$.

County of residence was associated with the utilisation of chemotherapy in the logistic regression analysis. Compared with the county of Troms, traditionally being an active county in the treatment of lung cancer, 15 counties had adjusted odds ratios $(\mathrm{OR})$ in the range of $0.13(95 \% \mathrm{CI} 0.1$ to 0.19$)$ to 0.52 (0.38 to 0.74$)$ for having a registered code, whereas three counties had OR in the range 0.94 (0.66 to 1.33$)$ to 1.04 (0.64 to 1.69). A later time of diagnosis (OR $1.28,95 \%$ CI 1.25 to 1.31 ) and radiation therapy (OR $3.13,95 \%$ CI 2.82 to 3.48 ) were also significantly associated with chemotherapy, whereas surgery (OR $0.53,95 \%$ CI 0.47 to 0.61 ), higher age (OR $0.61,95 \%$ CI 0.58 to 0.64 ) and male gender (OR $0.85,95 \%$ CI 0.77 to 0.94 ) were inversely associated.

\section{DISCUSSION}

The results of our study support the hypothesis that modern third-generation chemotherapy for ANSCLC is efficacious not 
Figure 3 Survival of patients with advanced non-small-cell lung cancer before and after the general introduction of third-generation chemotherapy in Norway.

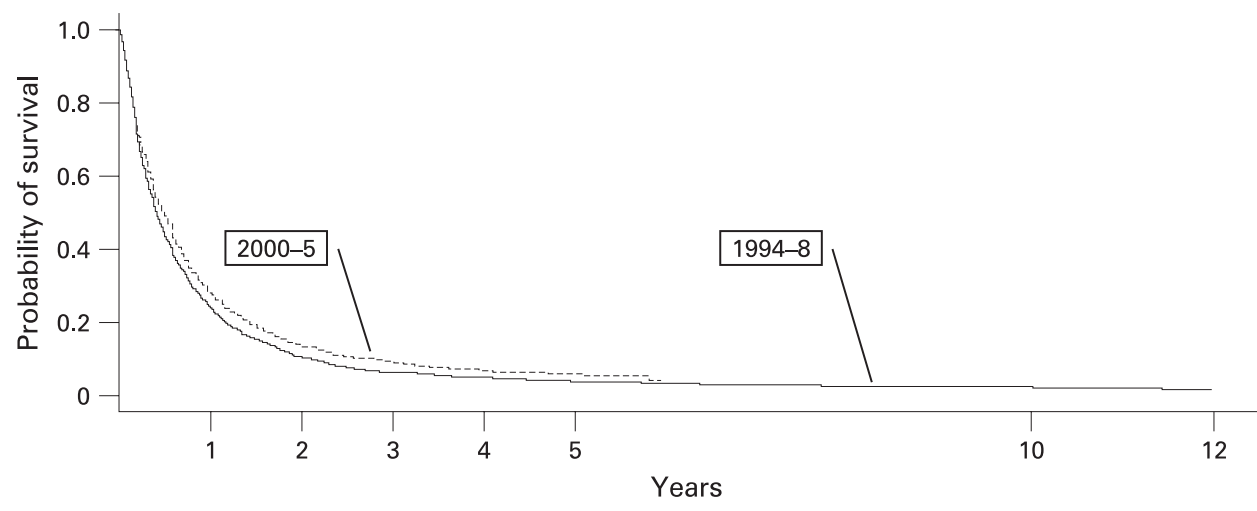

only in the setting of formal clinical trials but is also effective in current clinical practice at the population level. The use of thirdgeneration chemotherapy was associated with slightly increased survival in this large and non-selected population of patients with ANSCLC in Norway. Median survival increased by nearly 4 weeks and one-year survival increased by $4 \%$ after the introduction of third-generation chemotherapy. The amount of third-generation drugs used in the counties of Norway was positively correlated with survival in the ecological part of the study. In the cohort analysis, the survival of patients with registered chemotherapy was similar to the results of clinical trials. In all analyses, we found substantial geographical and temporal variations in chemotherapy use indicating deficiencies in the quality of delivered care.

The present study raises the question of whether the underuse of chemotherapy may be partly responsible for the comparatively low survival rates in Norway. One year survival of all Norwegian patients with lung cancer was $29 \%$ in 2003, higher than in the United Kingdom but lower than in Sweden. Spending in Euros for the indicator drug in the present study, vinorelbine, and for gemcitabine was up to twofold higher in Sweden than in Norway. Moreover, the introduction of thirdgeneration drugs lagged behind in Norway (fig 4), where vinorelbine was launched up to 10 years later than in other
Figure 4 Uptake of vinorelbine and (A) gemcitabine (B) (Euros per 100000 inhabitants) in Norway, Sweden and the United Kingdom (based on data from IMS LifeCycle and WHO Statistical Information System).
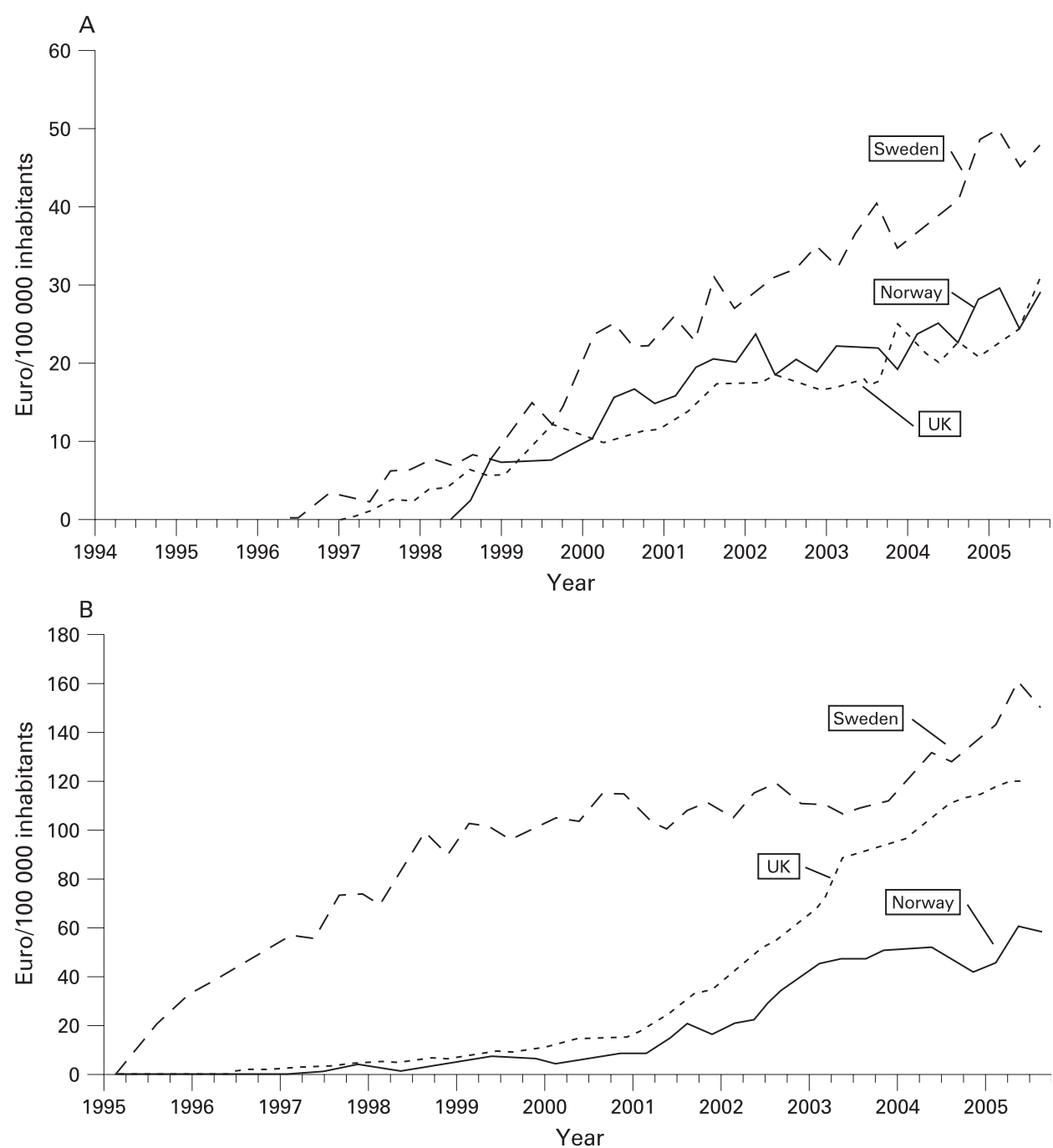
European countries. In a recent study, medical innovation represented by new drug launches for a variety of medical conditions was positively correlated with longevity. ${ }^{30}$ Similarly, the mean vintage of cancer drugs in the United States (FR Lichtenberg, unpublished data, 2007) and Europe ${ }^{31}$ was positively correlated with cancer survival rates. Third-generation cytotoxic drugs constituted an innovation of lung cancer treatment that took several years to reach patients in Norway. We are well aware that causal inferences regarding the effectiveness of chemotherapy cannot be drawn from the present study because of its ecological design. Furthermore, differences in survival between countries can arise from patientrelated factors, for example co-morbidities and tumour aggressiveness $^{32}$ as well as dissimilarities in cancer registries. On the other hand, the Nordic populations and cancer registries ${ }^{33}$ are very similar and the results of our analyses consistently indicate associations between the utilisation of chemotherapy and survival. We thus cannot rule out that the lower survival of patients in Norway can partly be attributed to the delayed use and underuse of third-generation chemotherapy.

The use of chemotherapy varied considerably across the 19 counties in Norway, a finding that is in accordance with earlier studies regarding variation within ${ }^{21} 233435$ and across $^{36}$ countries. We were nevertheless surprised by the high degree of variation in a country with only 4.7 million inhabitants and approximately 2300 cases of lung cancer annually, treated at 52 hospitals. Possible reasons for the variations include professional traditions and unclear or lacking national guidelines.

We regard vinorelbine a valid indicator for the use of thirdgeneration chemotherapy against ANSCLC in Norway. Its broader introduction during 1998-9 marked a transition from a generally more nihilistic attitude regarding the treatment of ANSCLC towards a more active approach. The treatment was gradually adopted throughout the country: in 1999, 12 out of 19 Norwegian counties used vinorelbine whereas in 2000 all counties used the drug. Clearly, the utilisation of vinorelbine for ANSCLC was higher than that of other third-generation drugs. The main driver for the introduction and predominant use of vinorelbine in Norway was most likely the first national chemotherapy protocol for ANSCLC that included nearly one quarter of potential patients during the years 2000-2. ${ }^{37}$ In 1999, 12 out of 19 counties used vinorelbine, whereas in 2000 all counties used the drug. Therefore, we suggest that vinorelbine is one valid indicator for the use of third-generation chemotherapy against ANSCLC in Norway. Although vinorelbine was a valid indicator in the particular context of the period of our study in Norway, however, other equally effective drugs, eg, gemcitabine or paclitaxel, ${ }^{14}$ probably have similar effects on survival from a population perspective.

Our study has certain limitations. First, we could not exclude patients with TNM stage IIIA who have a better prognosis, because only condensed TNM was available. This subgroup of patients is, however, small and their inclusion is unlikely to have influenced the overall results of this large population.

Second, technological advances in diagnostic procedures are a likely confounder of our results. In fact, we observed substantial stage shift in our study population, which probably implies that more patients are being diagnosed with asymptomatic, but more advanced, disease, which in turn would contribute to better prognosis in the advanced disease group of patients. Nevertheless, the influence of chemotherapy was maintained when we added annual rates of advanced disease and histological confirmation in a county as proxies for improved diagnostics to our Cox models (data not shown).
Third, we could not adjust for PS and co-morbidity because such clinical information was not registered. These factors are potential confounders of the before/after comparison but geographical variations are less likely. Anyhow, the inclusion in our study of patients with PS 3 and 4 and high co-morbidity, who usually do not receive chemotherapy, would attenuate associations between survival and chemotherapy utilisation.

Fourth, we were unable to assess QoL or treatment-related side effects, which obviously are crucial dimensions of palliative chemotherapy, because CRN does not collect these data. In most clinical trials of chemotherapy versus supportive care, dimensions of QoL have been congruent with survival outcomes $^{9-13}$ or stable ${ }^{8}$ under chemotherapy, but this is not necessarily the case in our less selected patient cohort.

Fifth, there are deviations from the proportional hazard assumption in most of our survival analyses, therefore the HR reported have to be interpreted as overall group differences.

Finally, improvements in supportive care, information on which was not available in the registry, are likely to have affected patients' survival positively after the introduction of chemotherapy. Like the use of cytotoxic drugs, the quality and intensity of supportive care can vary among healthcare institutions, ${ }^{38}$ whereas regional differences in a country with a uniform healthcare system are less likely. We therefore assume that improvements in supportive care contribute marginally to regional variations in survival.

In spite of these limitations, our observations appear valid and representative for the ANSCLC population and the healthcare system in Norway. We also believe that the results from this large study are applicable to other industrialised countries, with comparable populations and healthcare systems.

The quality of healthcare systems can be improved through "(...) changes that lead toward better patient and population outcomes (health), better system performance (care) and better professional development (learning)". ${ }^{39}$ The present study indicates improvement potential in all three areas. First, survival of patients with ANSCLC in Norway is lower than in some other countries (outcome). Second, the use of third-generation chemotherapy varied substantially (system performance). Third, lack of professional development and coordination may explain some of the observed variations (learning). Practice guidelines, professional networks, study protocols with broad patient inclusion, reporting of outcomes and benchmarking are potential tools to initiate the changes that lead to the faster and more homogenous uptake of evidence-based treatment options such as third-generation chemotherapy and thus improve care for patients with ANSCLC.

Acknowledgements: The authors greatly appreciate the expert assistance of the staff of the Cancer Registry of Norway and the National Institute of Public Health. They are grateful to Frank Jørgensen, Haukeland Hospital Pharmacy, for advice and data on chemotherapy utilisation and wish to thank Sidsel Kristiansen, the Hospital Pharmacy of North Norway Trust, Liv Reisch, the Hospital Pharmacy in Trondheim, Randi Myhr, Aker Hospital Pharmacy and Mette Thorvaldsen, the Hospital Pharmacy in Kristiansand for supplying data on chemotherapy utilisation at their hospitals.

Funding: $\mathrm{C} v \mathrm{P}$ has received scholarships from the Faculty of Medicine, University of Bergen as well as from the Western Norway Regional Health Authority and the Department of Thoracic Medicine, Haukeland University Hospital.

Competing interests: $\mathrm{C} v \mathrm{P}$ has received support for travel to scientific conferences from Eli Lilly, Astra, Roche, Glaxo, and research grants from the Regional Health Authority of Western Norway and from the University of Bergen. He chairs the protocol committee of an ongoing clinical trial that has received an unrestricted grant from Pierre Fabre, the manufacturer of Navelbine (vinorelbine). N W has received research grants from European Union, Karolinska Institute, Roche, Stockholm Cancer Foundation and Stockholm County Counsel. He was an employee at Bristol Myers Squibb during 2001-3. S Sundstrom has received travel support for scientific conferences from Lilly, 
Roche, Novartis, Pierre Phabre and AstraZeneca. He has received an unrestricted grant from Eli Lilly for a study in 2005. S Sörenson received unrestricted grants for academic studies from Pierre Fabre, Aventis and Pfizer and support for travel to ASCO and IASCLC meetings from Roche, Pfizer, and Eli Lilly. The other authors have no conflicts of interest.

Ethics approval: The study was approved by the Regional Ethical Committee, Bergen, Norway and by the data protection authority (NSD).

\section{REFERENCES}

1. Ferlay J, Autier $\mathrm{P}$, Boniol $\mathrm{M}$, et al. Estimates of the cancer incidence and mortality in Europe in 2006. Ann Oncol 2007;18:581-92.

2. Jemal A, Siegel R, Ward E, et al. Cancer statistics, 2007. CA Cancer J Clin 2007; 57:43-66.

3. Cancer Survival: England 1998-2003. National Statistics website: www. statistics.gov.uk 2007 http://www.statistics.gov.uk/STATBASE/ssdataset. asp?vInk = 8982 (accessed 23 May 2007).

4. Talbäck M, Rosen M, Stenbeck M, et al. Cancer patient survival in Sweden at the beginning of the third millennium-predictions using period analysis. Cancer Causes Control 2004;15:967-76.

5. Socinski MA, Morris DE, Masters GA, et al. Chemotherapeutic management of stage IV non-small cell lung cancer. Chest 2003;123(1 Suppl):226S-43S.

6. Pfister DG, Johnson DH, Azzoli CG, et al. American Society of Clinical Oncology treatment of unresectable non-small-cell lung cancer guideline: update 2003. J Clin Oncol 2004;22:330-53.

7. Spiro SG, Porter JC. Lung cancer-where are we today? Current advances in staging and nonsurgical treatment. Am J Respir Crit Care Med 2002;166:1166-96.

8. Brown J, Thorpe H, Napp V, et al. Assessment of quality of life in the supportive care setting of the Big Lung Trial in Non-Small-Cell Lung Cancer. J Clin Oncol 2005;23:7417-27.

9. Anderson H, Hopwood P, Stephens RJ, et al. Gemcitabine plus best supportive care (BSC) vs BSC in inoperable non-small cell lung cancer-a randomized trial with quality of life as the primary outcome. UK NSCLC Gemcitabine Group. Non-Small Cell Lung Cancer. Br J Cancer 2000;83:447-53.

10. Cullen MH, Billingham LJ, Woodroffe CM, et al. Mitomycin, ifosfamide, and cisplatin in unresectable non-small-cell lung cancer: effects on survival and quality of life. J Clin Oncol 1999:17:3188-94.

11. Helsing $\mathbf{M}$, Bergman $B$, Thaning $L$, et al. Quality of life and survival in patients with advanced non-small cell lung cancer receiving supportive care plus chemotherapy with carboplatin and etoposide or supportive care only. A multicentre randomised phase III trial. Joint Lung Cancer Study Group. Eur J Cancer 1998;34:1036-44.

12. Thongprasert S, Sanguanmitra P, Juthapan W, et al. Relationship between quality of life and clinical outcomes in advanced non-small cell lung cancer: best supportive care (BSC) versus BSC plus chemotherapy. Lung Cancer 1999;24:17-24.

13. Anonymous. Non-small Cell Lung Cancer Collaborative Group. Chemotherapy for non-small cell lung cancer. Issue 2. Cochrane Database Syst Rev 2000;2: doi:10.1002/14651858.CD002139.

14. Schiller JH, Harrington D, Belani CP, et al. Comparison of four chemotherapy regimens for advanced non-small-cell lung cancer. N Engl J Med 2002;346:92-8.

15. Vardy JL, Boyer ML, Beale P, et al. Patients in advanced non small cell lung cancer (ANSCLC) chemotherapy trials are not representative of the general lung cancer population [abstract]. Proc Am Soc Clin Oncol 2003;22:663.

16. Hutchins LF, Unger JM, Crowley JJ, et al. Underrepresentation of patients 65 years of age or older in cancer-treatment trials. N Engl J Med 1999;341):2061-7.

17. Murthy VH, Krumholz HM, Gross CP. Participation in cancer clinical trials: race-, sex-, and age-based disparities. JAMA 2004;291:2720-6.
18. Hotta K, Fujiwara Y, Matsuo K, et al. Recent improvement in the survival of patients with advanced non-small cell lung cancer enrolled in phase III trials of first-line, systemic chemotherapy. Cancer 2007;109:939-48.

19. Wakelee HA, Bernardo $\mathrm{P}$, Johnson $\mathrm{DH}$, et al. Changes in the natural history of nonsmall cell lung cancer (NSCLC) — comparison of outcomes and characteristics in patients with advanced NSCLC entered in Eastern Cooperative Oncology Group trials before and after 1990. Cancer 2006;106:2208-17.

20. Institute of Medicine. Crossing the quality chasm: a new health care system for the 21st century. Washington, DC: National Academy Press, 2001

21. Earle CC, Venditti LN, Neumann PJ, et al. Who gets chemotherapy for metastatic lung cancer? Chest 2000;117:1239-46.

22. Gregor A, Thomson CS, Brewster DH, et al. Management and survival of patients with lung cancer in Scotland diagnosed in 1995: results of a national population based study. Thorax 2001;56:212-17.

23. Jack RH, Gulliford MC, Ferguson J, et al. Geographical inequalities in lung cancer management and survival in South East England: evidence of variation in access to oncology services? Br J Cancer 2003;88:1025-31.

24. Emanuel EJ, Young-Xu Y, Levinsky NG, et al. Chemotherapy use among Medicare beneficiaries at the end of life. Ann Intern Med 2003;138:639-43.

25. Earle CC, Tsai JS, Gelber RD, et al. Effectiveness of chemotherapy for advanced lung cancer in the elderly: instrumental variable and propensity analysis. J Clin Oncol 2001;19:1064-70.

26. Ramsey SD, Howlader N, Etzioni RD, et al. Chemotherapy use, outcomes, and costs for older persons with advanced non-small-cell lung cancer: evidence from surveillance, epidemiology and end results-Medicare. J Clin Oncol 2004:22:4971-8.

27. Cartman ML, Hatfield AC, Muers MF, et al. Lung cancer: district active treatment rates affect survival. J Epidemiol Community Health 2002;56:424-9.

28. R: a language and environment for statistical computing organization. $R$ Foundation for Statistical Computing, 2007.

29. Harrel FE Jr. Regression modelling strategies with applications to linear models, logistic regression and survival analysis. New York: Springer, 2007

30. Lichtenberg FR. The impact of new drug launches on longevity: evidence from longitudinal, disease-level data from 52 countries, 1982-2001. Int J Health Care Finance Econ 2005:5:47-73.

31. Jönsson B, Wilking N. The effect of cancer drug vintage on cancer survival and mortality. Ann Oncol 2007;18(Suppl 3):iii67-77.

32. Imperatori A, Harrison RN, Leitch DN, et al. Lung cancer in Teesside (UK) and Varese (Italy): a comparison of management and survival. Thorax 2006;61:232-9.

33. Michelsen Lung E, Haunstrup Clemmensen I, Storm HH. Survey of Nordic Cancer Registries. Danish Cancer Society 2008 http://www.ancr.nu/survey.asp laccessed 2 Mar 2008).

34. Patel N, Adatia R, Mellemgaard A, et al. Variation in the use of chemotherapy in lung cancer. Br J Cancer 2007:96:886-90.

35. Mahmud SM, Reilly M, Comber H. Patterns of initial management of lung cancer in the Republic of Ireland: a population-based observational study. Lung Cancer 2003:41:57-64.

36. Jönsson B, Wilking N. Market uptake of new oncology drugs. Ann Oncol 2007:18(Suppl 3):iii31-48.

37. von Plessen C, Bergman B, Andresen 0 , et al. Palliative chemotherapy beyond three courses conveys no survival or consistent quality-of-life benefits in advanced nonsmall-cell lung cancer. Br J Cancer 2006:95:966-73.

38. Wennberg JE, Fisher ES, Stukel TA, et al. Use of hospitals, physician visits, and hospice care during last six months of life among cohorts loyal to highly respected hospitals in the United States. BMJ 2004;328:607-11.

39. Batalden PB, Davidoff F. What is "quality improvement" and how can it transform healthcare? Qual Saf Health Care 2007:16:2-3.

\section{Access a vast information database with Toll-Free linking}

"Toll-free" linking gives you immediate access to the full text of many of the cited articles in a paper's reference list-FOR FREE. With the support of HighWire's vast journal catalogue, a huge reference library is now open to you. If HighWire hosts the journal, you can view the full text of the referenced article, completely free of charge by following the Free Full Text links in the references. 